\title{
Food group intakes of a representative sample of pre-school children in Ireland
}

\author{
K. Evans, J. Walton and A. Flynn \\ School of Food and Nutritional Sciences, University College Cork, Cork, Republic of Ireland
}

The objective of this study was to determine food and beverage intakes of Irish pre-school children using data from the National Pre-School Nutrition Survey (NPNS) (2010-2011). A 4 day weighed dietary record was used to collect food and beverage intake data from a representative sample of 500 children aged 1-4 years in Ireland. The study is described in more detail on the Irish Universities Nutrition Alliance (IUNA) website ${ }^{(1)}$.

\begin{tabular}{|c|c|c|c|c|c|c|c|c|}
\hline \multicolumn{9}{|c|}{$\begin{array}{cc}\text { daily food group intakes }(\mathrm{g} / \mathrm{d}) \text { of Irish pre-school children aged } 1-4 \text { years (total population) } \\
1 \mathrm{yr}(n=126) & 2 \mathrm{yr}(n=12\end{array}$} \\
\hline & $\overline{\text { Mean }}$ & $\mathrm{SD}$ & Mean & $\overline{\mathrm{SD}}$ & Mean & $\overline{\mathrm{SD}}$ & Mean & SD \\
\hline \multicolumn{9}{|l|}{ Dairy products } \\
\hline Whole milk & 283 & 228 & 231 & 223 & 189 & 169 & 172 & 148 \\
\hline Reduced fat milk & 16 & 78 & 32 & 92 & 37 & 101 & 52 & 112 \\
\hline Infant/Growing up milk & 113 & 200 & 56 & 137 & 13 & 63 & 6 & 46 \\
\hline Yoghurt and fromage frais & 62 & 44 & 60 & 57 & 65 & 62 & 64 & 50 \\
\hline Cheese & 8 & 10 & 9 & 10 & 8 & 11 & 10 & 12 \\
\hline \multicolumn{9}{|l|}{ Meat } \\
\hline Fresh meat & 7 & 9 & 11 & 15 & 12 & 14 & 15 & 18 \\
\hline Processedlcured meat & 16 & 22 & 25 & 23 & 30 & 23 & 34 & 29 \\
\hline Meat dishes & 43 & 44 & 38 & 52 & 32 & 40 & 33 & 42 \\
\hline \multicolumn{9}{|l|}{ Bread } \\
\hline White bread & 13 & 17 & 21 & 25 & 29 & 26 & 34 & 28 \\
\hline Wholemeallbrown bread & 10 & 15 & 21 & 24 & 19 & 21 & 20 & 24 \\
\hline \multicolumn{9}{|l|}{ Breakfast cereals } \\
\hline Ready-to-eat breakfast cereal & 13 & 11 & 18 & 14 & 22 & 15 & 25 & 18 \\
\hline Other breakfast cereal & 23 & 39 & 22 & 41 & 21 & 47 & 15 & 36 \\
\hline \multicolumn{9}{|l|}{ Fruit and vegetables } \\
\hline Whole fruit & 115 & 74 & 134 & 87 & 139 & 99 & 132 & 88 \\
\hline Fruit juices (100\% fruit) & 23 & 50 & 38 & 61 & 65 & 82 & 77 & 99 \\
\hline Vegetables & 29 & 32 & 29 & 28 & 31 & 27 & 39 & 32 \\
\hline \multicolumn{9}{|l|}{ Beverages (excluding milk) } \\
\hline Water (as a beverage) & 129 & 146 & 166 & 207 & 137 & 133 & 133 & 142 \\
\hline Soft drinks (not low calorie) & 21 & 66 & 49 & 103 & 52 & 91 & 77 & 181 \\
\hline Soft drinks (low calorie) & 68 & 144 & 103 & 181 & 104 & 156 & 111 & 177 \\
\hline Biscuits, cakes, confectionery \& savoury snacks & 17 & 16 & 30 & 23 & 34 & 19 & 43 & 24 \\
\hline
\end{tabular}

There is a notable change in dietary patterns with increasing age from age 1- 4 years. By age 4, dietary preferences and food habits which could lead to nutrient imbalance have emerged, such as, intake of whole milk instead of reduced fat milk, processed meats instead of fresh meat and white bread rather than brown/wholemeal. Intakes of soft drinks, fruit juices, biscuits and confectionery increased with increasing age. These findings will be useful in the development of food based dietary guidelines for pre-school children.

The project was funded by the Department of Agriculture, Food and the Marine under the "Food for Health Research Initiative" (FHRI) 2007-2012

1. Irish Universities Nutrition Alliance (IUNA) (2012) National Pre-School Nutrition Survey- Summary Report. Available online at www.iuna.net 